\title{
EVALUATION OF ORAL SPLINT IN TREATMENT OF TEMPROMANDIBULAR DISORDERS IN CLASS I MALOCCLUSION
}

\author{
Hussein N. Al-Khalifa*
}

\begin{abstract}
Objective: This study was designed to evaluate the effect of occlusal splints used for treatment of tempromandibular disorders (TMD).

Material and methods: Thirty class I malocclusion patients (ranging in age 20-30) suffering from signs of TMD attending at orthodontic department, faculty of dental medicine, Al Azhar university boys branch. The patients' complaints were crowding of teeth, limited mouth opening, tempromandibular joints (TMJ) pains, and joint noises during function. The patients will be divided randomly into three groups. Group I will have patients treated by hard rigid occlusal splint (heat cured tooth borne and occlusal surface). Group II will have patients treated by soft occlusal splint flexible and pliable tooth borne and occlusal surface. Group III will have patients treated by dual laminated occlusal surface, hard acrylic resin and soft material on tooth borne surface. Clinical evaluation was performed before the delivery of the splint and at 2, 4, 6 months. Maximum mouth opening and joint sound were recorded each follow up visit.
\end{abstract}

Results: All groups of the study showed improvement in maximal mouth opening, TMJ pain, and clicking sounds with variable degrees according to treatment method.

Conclusion: Dual laminated occlusal splint seemed to be superior in achieving rapid and prolonged improvement in TMD patients than other splints.

KEY WORDS: Oral splint, tmd ,class I malocclusion

\section{INTRODUCTION}

The Tempromandibular joint (TMJ) is the joint between the lower jaw and the base of the skull. Tempromandibular joint dysfunction (TMD) is a clinically significant condition in orthodontic patients which can be a source of acute or chronic orofacial pain and dysfunction including limitation of movement causing pain, clicking, crepitus and deviating jaw function and joint sounds. ${ }^{(1)}$ Anderson et al. reported that approximately $75 \%$ of the population having at least one sign of joint

\footnotetext{
* Associate Professor of Orthodontics, Al-Azhar University, Faculty of Oral and Dental Medicine (Boys Branch),
} Cairo, Egypt 
dysfunction (abnormal jaw movement, tenderness on palpation, joint noises, etc.). ${ }^{(2)}$

The primary goal of treatment of TMD is to reduce pain and jaw dysfunction. TMD treatment can be divided into; conservative methods and surgical methods. Conservative treatment includes medication, habit modification, counseling, physical therapy, splint therapy, and manipulation. ${ }^{(3,4)}$ Surgical treatment includes, arthocentesis, arthroscopy, arthroplasty, dissectomy, and TMJ reconstruction. ${ }^{(5)} \mathrm{A}$ conservative reversible method is a widely accepted to be first choice for treatment of TMD as one of conservative treatment, occlusal splints has been frequently used for treatment of TMD. ${ }^{(6)}$

Occlusal adjustment involves repositioning the mandible to a centric position by using prosthodontic or orthodontic appliances, intraoral occlusal splints are designed to provide even a balanced occlusal contact without forcefully altering the mandibular rest position or permanently the dental occlusion. Occlusal splint made of processed hard acrylic splints worn on the teeth like retainer or removable denture. ${ }^{(7)}$ This splint is often successful, but the length of time required to reach a pain-free normal range of motion is sub-optimum. ${ }^{(8)}$

Occlusal splint is defined as a removable artificial occlusal surface used for diagnosis or therapy affecting the relationship of the mandible to the maxilla. Occlusal appliances may be used in different ways for occlusal stabilization, prevent wear of the dentition, or for treatment of TMJ disorders. There are different types of occlusal appliances, each one has its special design, indication and precautions that should be followed so that clinicians strongly advised to thoroughly understand the masticatory system dynamics and perform a comprehensive examination to the TMJ and its related structures to be able to choose correct appliance perfectly with fewer complications. Based on literature, occlusal appliances were originally made from acrylic resin and cover all or most of the teeth in one arch. The mechanisms of action of the occlusal appliances as a treatment include occlusal disengagement, restoring vertical dimension of occlusion, muscles relaxing, joint unloading, or TMJ repositioning. ${ }^{(9)}$

Occlusal splints are used like stabilization splint, modified Hawley splint, and repositioning splint for the treatment of TMDs patients. Soft splints, which are more convenient for patients than hard splint, can be used immediately after provisional diagnosis of TMD. The rationale for using soft splints is that the soft resilient material may help in distributing the heavy load associated with Para functional Habits. Hard splints are thought to reduce TMD symptoms by altering the occlusal equilibrium, changing the afferent impulses to the central nervous system, improving the vertical dimension, correcting the condylar position, and aiding cognitive awareness. These splints could be made to fit on either the maxillary or mandibular arch features, but maxillary splints are better options. Occlusal splints works on the principle of Muscles relaxing, unloading of the joint, repositioning of the joint, occlusal disengagement and restoration of the occluso vertical dimension. ${ }^{(10)}$

There are three different main types of the materials of splints; 1) hard rigid acrylic resin occlusal splints, 2) soft resilient acrylic resin occlusal splint, and 3) dual laminated hard acrylic resin lined by soft acrylic resin. The dual type combines the advantages of both soft and hard acrylic resins. In addition, there are several types of oral appliances; 1) Muscle relaxation appliance 2) Anterior repositioning appliances 3) Anterior bite plane 4) Pivoting appliance 5) Neuromuscular appliance 6) Hydrostatic appliance. ${ }^{(11)}$

Muscle relaxation appliance, also known as Flat plane stabilization appliance, provides joint stabilization, protect the teeth, redistribute the occlusal forces, relax the elevator muscles, decrease bruxism, increases the patient's awareness of jaw habits and helps alter the rest position of the mandible to a more relaxed, open position. ${ }^{(12)}$

Macro and Micro anterior bite plane are designed 
as a palatal-coverage horseshoe shape with an occlusal table covering 6 to 8 anterior maxillary teeth or only 2 to 4 anterior teeth. The purpose of this appliance is to disengage the posterior teeth, thus eliminating the influences of the posterior occlusion on the masticatory system. ${ }^{(13,14)}$

Pivot appliances are made of hard acrylic resin that covers the maxillary arch with a single posterior occlusal contact that may be bilateral or unilatral, placed as far posteriorly as possible, in each side. It reduces intra-articular pressure by unloading of the articular surfaces of the joint. The pivot appliance is used mainly internal derangement or with osteoarthritis adverse effect with use of unilateral appliance is posterior open bite in pivot area. ${ }^{(15)}$

Neuromuscular Appliances (NMA) Neuromuscular dentistry (NMD) have Advocated that by use of jaw muscle stimulators with jaw-tracking machines to produce an occlusal appliance that is at the ideal vertical and horizontal position of the mandible in relation to the cranium.

While posterior bite plane appliances are made to be worn on the lower arch. The design consist of a bilateral hard acrylic resin table, creates a disocclusion of the anterior teeth, located over the mandibular molars and premolars and connect with a lingual metal bar. These appliances intended to produce vertical dimension and horizontal maxillomandibular relationship changes. ${ }^{(16)}$ Hydrostatic appliance is designed by Lerman in the form of bilateral waterfilled plastic chamber attached to acrylic palatal appliance and patients' posterior teeth occluded with water filled chambers. ${ }^{(17)}$

There are also several uses for occlusal splints:

1. Limiting the closure in maximal intercuspal position: The occlusal splint help the patient to place his mandible in a new posture, thus resulting in a new muscular and articular balance

2. Distribution of forces: The forces generated during bruxism can be as much as six times the maximal force generated by normal chewing.
They act by distributing these forces across the masticatory system

3. Normalizing periodontal ligament proprioception: An occlusal splint balances the load and allows for muscle symmetry by dissipating the forces placed on individual teeth by utilizing a larger surface area covering all teeth in the arch

4. Relaxing the muscles: A splint will relax the elevator and positioning muscles, with immediate disclusion of all posterior teeth by the anterior guidance and condylar guidance in all movements

5. Allowing the condyles to seat in centric relation: A properly balanced splint allows the articulator disc to obtain its antero-superior position over the condylar head, resulting in an occlusion associated with relaxed positioning and elevator muscles

6. Increase in the vertical dimension of occlusion: Occlusal splints can be adjusted with a vertical height that exceeds the physiologic interocclusal distance

7. Cognitive awareness theory: A patient can learn what position or activities are harmful, by increased cognitive awareness regarding the positioning and use of the jaw, the change in oral tactile stimuli, and the decrease in oral volume. $^{(18)}$

\section{Aim of the study}

The aim of the study was to evaluate the effect of the occlusal splint in the treatment of TMD.

\section{MATERIAL AND METHOD}

The study included thirty patients class I malocclusion with age ranging 20 - 30 years, suffering from signs of TMD at out patient clinic, orthodontic department, faculty of oral and dental medicine, Al Azhar University. The patient complaints were limited mouth opening, TMJ pain and joints noises during functions. 


\section{Patient grouping:}

The patients divided randomly into three groups;

1) Group I: Patients in this group treated with hard acrylic splint. (Fig.1)

2) Group II: Patients in this group treated with soft flexible splint. (Fig.2)

3) Group III: Patients in this group treated with dual laminated hard and soft splint. (Fig.3)

\section{Inclusion criteria:}

Male and female patients were diagnosed with TMD. Diagnostic criteria of TMD include a history of TMJ noise, limitation of movement, pain, and joint sounds.

\section{Exclusion criteria:}

- Patients with systemic disease as rheumatoid arthritis.

- Degenerative changes of condyle head.

- $\quad$ Truma of TMJ.

- Anomalies of face and jaw.

- History of surgery in TMJ.

\section{Clinical evaluation:}

Patients were seated in anatomical position 'head supported' in order to avoid movement from cervical vertebrae, then patients were asked for:

1) Maximum mouth opening: to measure the distance form the incisal edge of maxillary central incisor to the incisal edge of mandibular central incisors by a digital calliper.

2) TMJ palpation: for detection of any tender area at the joint by applying finger tips over TMJ on both sides.

3) TMJ sounds: using a stethoscope over the TMJ and ask the patient to open to the maximum and then to close, doing lateral movement and

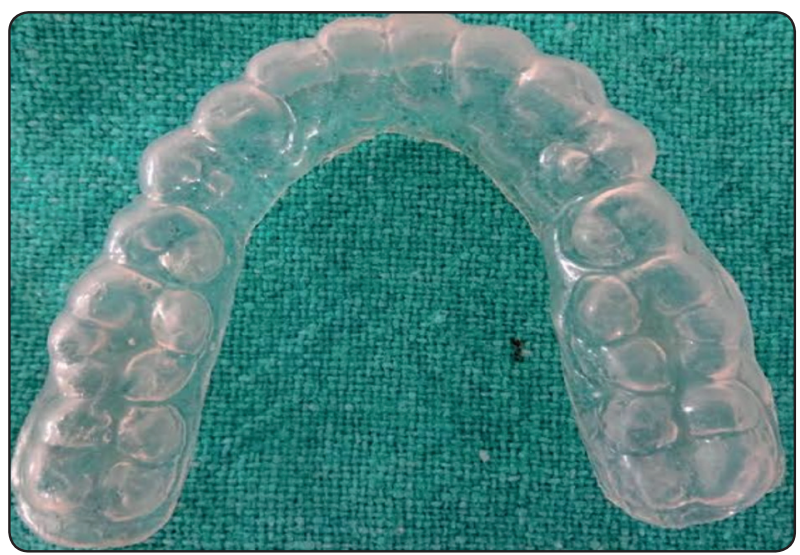

Fig. (2) Soft splint

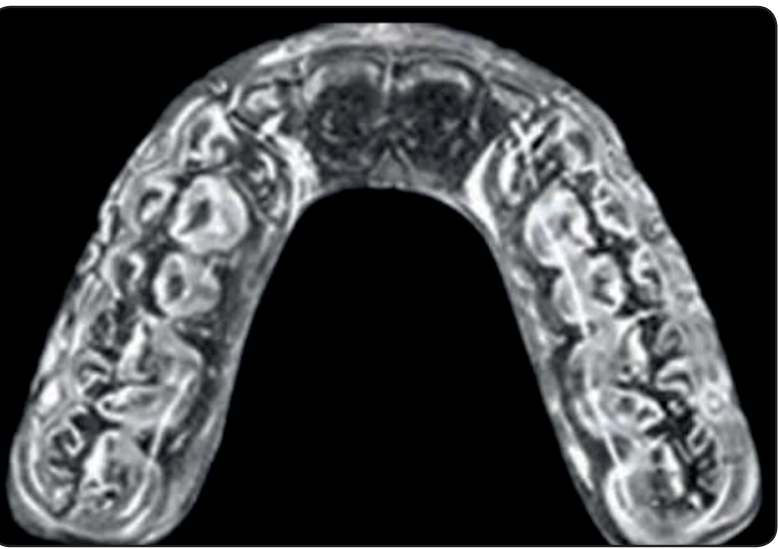

Fig. (1) Hard acrylic splint

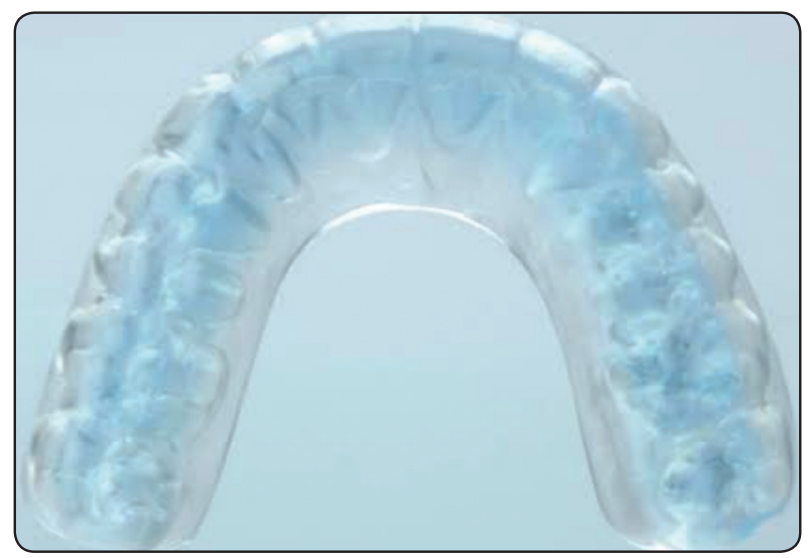

Fig. (3) Dual laminated splint protrusion and retrusion movement. 


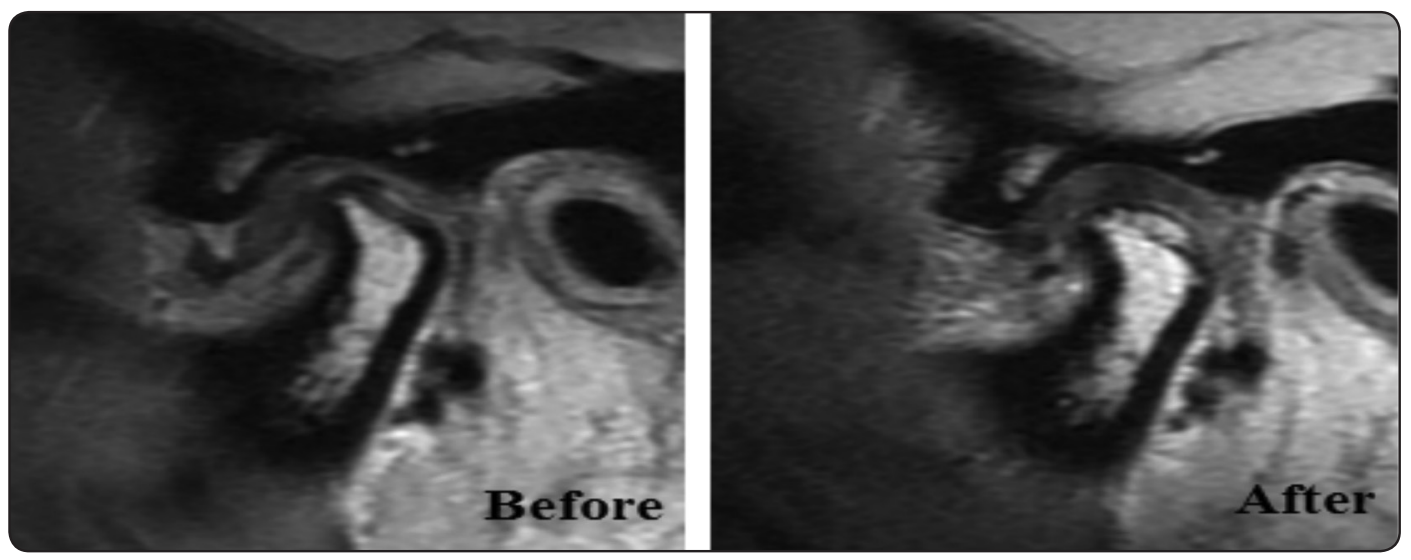

Fig. (4) MRI before and after splint therapy.

4) Muscle palpation: by the detection whether there is any pain or tenderness on temporails, masseter, internal and external pterygoid, trapezius and sternocleidomastoid muscle.

5) Visual analogue scale: all patients had been examined (Vas $10-\mathrm{cm}$ line), which ranged from 0 (no pain) to 10 (the worst possible pain). Clinical examination was performed before treatment and at: day 1,2 months, 4 months, and 6 months after splints' treatment.

\section{Imaging Evaluation:}

Magnetic Resonance Imaging (MRI) was taken to dental disc position and abnormalities of TMJ. MRI images were taken 2 times before and 6 months after treatment. A total of 30 patients with TMD were enrolled in this study. All patients were followed for six months and three were evaluated in this study visual Analogue Scale (VAS), maximum mouth opening (MMO) and joint sound (JS). (Fig. 4)

\section{RESULTS}

A total of 30 patients with pain from TMD and joint sounds were included in this study. Ten patients were treated with soft splints, other ten patients were treated with hard splints and the last
10 patients were treated with dual laminated splint. All patients were followed for 6 months. After tow months, gradual improvement in pain, maximum mouth opening and joint sounds were noticed in all groups especially group three that the other groups. Three parameters were evaluated in the current study (VAS), (MMO) and (JS).

Regarding the pain measurement score, table I shows low improvement significant level in group I, moderate improvement significant level in group II and high improvement significant level in group III as shown in table 1 .

MMO improved in all groups, but highly significant in group III than group I and II. Group III showed immediate and prolonged improvement than the other two groups as shown in Table 2.

As regard to joints sounds, improvement across observation time from two months till six months especially in group III. No improvement significant level in group I, low improvement significant level in group II and high improvement significant level in group III as shown in table 3 .

Data were analyzed using Statistical Program for Social Science (SPSS) version 20.0 Quantitative data were expressed as mean \pm standard deviation (SD). Qualitative data were expressed as frequency and percentage. 
TABLE (1) Visual Analogue Scale (VAS) score

\begin{tabular}{|c|c|c|c|c|c|}
\hline \multicolumn{2}{|c|}{ VAS } & Day 1 & Month 2 & Month 4 & Month 6 \\
\hline \multirow{3}{*}{ G I } & Mean & 7.00 & 5.14 & 3.79 & 3.07 \\
\hline & S.D & 0.96 & 0.94 & 0.80 & 0.47 \\
\hline & \multicolumn{2}{|c|}{$\mathrm{p}$ value } & $0.000 *$ & $0.0000 *$ & $0.0000 *$ \\
\hline \multirow{3}{*}{ G II } & Mean & 6.43 & 2.57 & 2.50 & 2.71 \\
\hline & S.D & 1.28 & 0.72 & 0.65 & 0.47 \\
\hline & \multicolumn{2}{|c|}{$\mathrm{p}$ value } & $0.0000^{*}$ & $0.0000^{*}$ & $0.0000^{*}$ \\
\hline \multirow{3}{*}{ G III } & Mean & 6.36 & 2.29 & 1.64 & 1.21 \\
\hline & S.D & 1.01 & 0.73 & 0.50 & 0.43 \\
\hline & \multicolumn{2}{|c|}{$\mathrm{p}$ value } & $0.0000^{*}$ & $0.0000^{*}$ & $0.0000 *$ \\
\hline$F$ test & & 1.453 & 56.333 & 37.205 & 65.114 \\
\hline$P$ value & & 0.246 & $0.001^{*}$ & $0.001 *$ & $0.001^{*}$ \\
\hline $\mathrm{P} 1$ & & 0.175 & $0.001 *$ & $0.001^{*}$ & $0.045^{*}$ \\
\hline $\mathrm{P} 2$ & & 0.128 & $0.001 *$ & $0.001 *$ & $0.001 *$ \\
\hline P3 & & 0.864 & 0.341 & $0.001 *$ & $0.001 *$ \\
\hline
\end{tabular}

P1: $G I \& G I I$

P2: $G I \& G I I I$

P3: G II \& G III

TABLE (2) Maximum mouth opening (MMO)

\begin{tabular}{|c|c|c|c|c|c|}
\hline \multicolumn{2}{|c|}{ MMO } & Day 1 & Month 2 & Month 4 & Month 6 \\
\hline \multirow{3}{*}{ G I } & Mean & 26.36 & 28.14 & 29.86 & 32.21 \\
\hline & S.D & 3.00 & 3.08 & 2.98 & 2.75 \\
\hline & \multicolumn{2}{|c|}{$\mathrm{p}$ value } & 0.127 & $0.004 *$ & $0.000^{*}$ \\
\hline \multirow{3}{*}{ G II } & Mean & 26.43 & 31.71 & 31.29 & 30.64 \\
\hline & S.D & 2.28 & 1.90 & 2.05 & 2.13 \\
\hline & \multicolumn{2}{|c|}{$\mathrm{p}$ value } & $0.001 *$ & $0.001 *$ & $0.001 *$ \\
\hline \multirow{3}{*}{ G III } & Mean & 27.50 & 32.93 & 33.36 & 34.07 \\
\hline & S.D & 3.41 & 2.40 & 2.06 & 2.09 \\
\hline & \multicolumn{2}{|c|}{$\mathrm{p}$ value } & $0.001 *$ & $0.001 *$ & $0.001 *$ \\
\hline F test & & 0.666 & 13.764 & 7.489 & 7.498 \\
\hline $\mathrm{P}$ value & & 0.520 & $0.001 *$ & $0.002 *$ & $0.002^{*}$ \\
\hline P1 & & 0.949 & $0.001 *$ & 0.124 & 0.084 \\
\hline $\mathrm{P} 2$ & & 0.309 & $0.001 *$ & $0.001 *$ & $0.043^{*}$ \\
\hline P3 & & 0.340 & 0.208 & $0.028 *$ & $0.001^{*}$ \\
\hline
\end{tabular}


TABLE (3) Joint sounds (JS)

\begin{tabular}{|c|c|c|c|c|c|c|c|c|c|}
\hline \multirow{2}{*}{\multicolumn{2}{|c|}{ Joint sounds }} & \multicolumn{2}{|c|}{ Day 1} & \multicolumn{2}{|c|}{ Month 2} & \multicolumn{2}{|c|}{ Month 4} & \multicolumn{2}{|c|}{ Month 6} \\
\hline & & & & & & & & & \\
\hline \multirow{4}{*}{ G I } & Constant & 10 & 100 & 7 & 70 & 7 & 70 & 8 & 80 \\
\hline & Improved & 0 & 0 & 1 & 10 & 1 & 10 & 0 & 0 \\
\hline & Occasional & 0 & 0 & 2 & 20 & 2 & 20 & 2 & 20 \\
\hline & \multicolumn{3}{|c|}{$P$ value } & \multicolumn{2}{|c|}{0.171} & \multicolumn{2}{|c|}{0.171} & \multicolumn{2}{|c|}{0.136} \\
\hline \multirow{4}{*}{ G II } & Constant & 10 & 100 & 9 & 90 & 7 & 70 & 4 & 40 \\
\hline & Improved & 0 & 0 & 1 & 10 & 2 & 20 & 4 & 40 \\
\hline & Occasional & 0 & 0 & 0 & 0 & 1 & 10 & 2 & 20 \\
\hline & \multicolumn{3}{|c|}{ P value } & \multicolumn{2}{|c|}{0.305} & \multicolumn{2}{|c|}{0.171} & \multicolumn{2}{|c|}{$0.014 *$} \\
\hline \multirow{4}{*}{ G III } & Constant & 10 & 100 & 6 & 60 & 5 & 50 & 3 & 30 \\
\hline & Improved & 0 & 0 & 2 & 20 & 3 & 30 & 5 & 50 \\
\hline & Occasional & 0 & 0 & 2 & 20 & 2 & 20 & 2 & 20 \\
\hline & \multicolumn{3}{|c|}{ P value } & \multicolumn{2}{|c|}{0.082} & \multicolumn{2}{|c|}{$0.036^{*}$} & \multicolumn{2}{|c|}{$0.005^{*}$} \\
\hline $\mathrm{P} 1$ & & & \multicolumn{2}{|c|}{0.325} & \multicolumn{2}{|c|}{0.717} & \multicolumn{2}{|c|}{0.069} \\
\hline $\mathrm{P} 2$ & & \multicolumn{2}{|c|}{-} & \multicolumn{2}{|c|}{0.815} & \multicolumn{2}{|c|}{0.513} & \multicolumn{2}{|c|}{$0.023 *$} \\
\hline P3 & & \multicolumn{2}{|c|}{ - } & \multicolumn{2}{|c|}{0.231} & \multicolumn{2}{|c|}{0.648} & \multicolumn{2}{|c|}{0.881} \\
\hline
\end{tabular}

\section{DISCUSSION}

There are different types of occlusal appliances, each one has its special design, indication and precautions that should be followed so that clinicians strongly advised to thoroughly understand the masticatory system dynamics and perform a comprehensive examination to the TMJ and its related structures to be able to choose correct appliance perfectly with fewer complications.

Conventional methods of splint fabrication are highly technique sensitive and often lead to poor splint fit, over contour, and hence poor patient compliance. Furthermore, patients often complain that they feel uncomfortable, too tight, or are too bulky to wear. They may also be self-conscious with wearing a cumbersome and obvious occlusal splint as reported by Dunn et al. ${ }^{(19)}$
Traditionally, splint products are made from polymethyl methacrylate (PMMA) and as a result are prone to fracture and failure during use. Splints are thought to unload the joint by disarticulating the dentition and increasing the vertical dimension of occlusion. By unloading the joint, there will be a reduction in both synovitis and masticatory muscle activity. ${ }^{(20)}$ Therefore, the result is a reduction in symptoms. These appliances may also change condylar position and the existing occlusal relationship, thereby reducing abnormal muscle activity and spasm as reported by Okeson. ${ }^{(21)}$

TMD can be treated through conservative and surgical interventions but conservative treatment is considered more beneficial. Conservative treatment includes splint therapy. ${ }^{(22)}$ Significant improvement in mouth opening was observed in the three groups. These results are comparable to Suvinen and 
Reade, ${ }^{(23)}$ who reported a $7.4 \mathrm{~mm}$ increase in jaw opening after splint therapy. All splint therapies relieved the painful muscular spasm and muscular tenderness leading to maximal jaw opening.

In the current study, significant reduction in the TMJ tenderness, muscular pain VAS score was observed in all splint groups. These observations were similar to the study done by Amin et al. ${ }^{(20)}$. They found that hard splints were more effective throughout the study period. These results were in contrast to the study of Tsuga et al. ${ }^{(24)}$ who found that hard splints were ineffective in reducing muscle pain. Reduction in muscle pain was due to reduction of abnormal muscle hyperactivity because occlusal splints contacts on all of the teeth, with immediate disclusion of all posterior teeth by the anterior teeth and condylar guidance. This will relax the elevator and positioning muscles. ${ }^{(20-24)}$ In regard to pain all groups showed improvement gradually from the first month till the six months of treatment with varying degree of improvement from patient with hard splint to soft splint to dual splint with the highest degree of significance with dual laminated splint.

In the present study, all splints were effective, these finindings were in accordance to the randomized trials of Amin et al. ${ }^{(20)}$ and Truelove et al. ${ }^{(25)}$ they found that all the patients improved irrespective of splint type, but in contrast to the study of Davies and Gray ${ }^{(26)}$ who found no advantage of any particular splint use.

In the current study, hard splints were effective than soft splints in contrast to the study by Pettengill et al. ${ }^{(27)}$ that reported no difference between both splints. In addition, the results of the current study are in accordance with Block et al. ${ }^{(28)}$ who concluded that almost $74 \%$ of patients with TMDs had complete remission of symptoms after 6 weeks of occlusal splint therapy but maximum improvement in study participants were observed in three months period.

\section{The following tests were done:}

- A one-way analysis of variance (ANOVA) when comparing between more than two means.

- Chi-square $\left(\mathrm{X}^{2}\right)$ test of significance was used in order to compare proportions between two qualitative parameters.

\section{CONCLUSION}

From the result of this study, it can be concluded that:

1. Dual laminated occlusal splints are superior in achieving rapid and prolonged improvement in TMD patients than other splints

2. All splints therapies are helpful in the improvement of TMDs.

3. Six months is considered to be an adequate time for splint therapy to improve symptoms.

4. Dual laminated and hard splint therapies showed earlier improvement of TMDs symptoms than soft splints.

\section{REFERENCES}

1. Peagle D, Holmund A, Hjerpe A. Expression of peotoglycan mrna in patients with painful clinking and chronic closed lock of tmj. Int j oral maxillofac surg. 2005; 34: 656-58.

2. Anderson G, Schiffman E, Schellhas K, Fricton J. "Clinical vs. arthrographic diagnosis of TMJ internal derangement.” Journal of dental research vol. 68,5 (1989): 826-9. doi:10.1177/00220345890680051501

3. Karlis V, Glickman R. Nonsurgical management of temporomandibular disorders. In: Miloro M, Ghali G, Larsen $\mathrm{P}$, Waite P. Peterson's principles of oral and maxillofacial surgery. 2nd ed. Hamilton: bc decker.2004 :949-50.

4. Ohnuki T, Fukuda M, Nakata A, Nagai H, Takahashi T, Sasano T. Evaluation of the position, mobility, and morphology of the disc by mri before and after four different treatments for temporomandibular joint disorders. Dentomaxillofac radiol.2006; 35:103-09.

5. Choi J, Kim I, Oh N, Oh S, Kim E, Lee S. A clinical study in the prognosis of the temporomandibular disorder. J korean assoc oral maxillofac surg. 2000;26:497-506. 
6. Hupp J, Ellis E, Tucker M. Contemporary Oral and Maxillofacial Surgery. 5th ed. St. Louis: Mosby. 2008: 617-650.

7. Seifeldin S. and Elhayes K. Soft versus hard occlusal splint therapy in the management of temporomandibular disorders (tmds). Saudi Dent J. 2015; 27: 208-14.

8. Lee H., Baek H., Song D, Kim H., Kim H., Kim B. et al. Effect of simultaneous therapy of arthrocentesis and occlusal splints on temporomandibular disorders: anterior disc displacement without reduction. J Korean Assoc Oral Maxillofac Surg. 2013; 39: 14-20.

9. Okeson JP (2007) Management of temporomandibular disorders and occlusion: Elsevier Health Sciences.

10. Dylina T.J. A common-sense approach to splint therapy. J. Prosthet. Dent. 2001;86:539-545.

11. Alqutaibi, A. Y., and A. N. Aboalrejal. "Types of occlusal splint in management of temporomandibular disorders (TMD).”J Arthritis 4.176 (2015): 2.

12. De Leeuw R, Pain O (2013) Guidelines for assessment, diagnosis, and management. (5th ed), Quintessence Publishing $\mathrm{Co}$, Inc, Chicago.

13. Klasser, Gary D, and Charles S Greene. "Oral appliances in the management of temporomandibular disorders." Oral surgery, oral medicine, oral pathology, oral radiology, and endodontics vol. 107,2 (2009): 212-23.

14. Jokstad A, Mo A, Krogstad BS. Clinical comparison between two different splint designs for temporomandibular disorder therapy. Acta Odontol Scand. 2005;63(4):218-226.

15. Ito, T., Gibbs, C., Marguelles-Bonnet, R., Lupkiewicz, S., Young, H., Lundeen, H., \& Mahan, P. "Loading on the temporomandibular joints with five occlusal conditions." The Journal of prosthetic dentistry vol. 56,4 (1986): 478-84.

16. Cooper BC. The role of bioelectronic instrumentation in the documentation and management of temporomandibular disorders. Oral Surg Oral Med Oral Pathol Oral Radiol Endod (1997) 83: 91-100.

17. Lerman MD. The hydrostatic appliance: a new approach to treatment of the TMJ pain-dysfunction syndrome. J Am Dent Assoc (1974) 89: 1343-1350.
18. Dawson PE. Evaluation, Diagnosis and Treatment of Occlusal Problems. 2nd ed. St. Louis, MO: Mosby; 1989.

19. Dunn DB, Michael F, Lewis B. CAD/CAM Occlusal splints: A new paradigm. Australas Dent Pract 2011;22:131.

20. Amin A, Meshramkar R, Lekha K. Comparative evaluation of clinical performance of different kind of occlusal splint in management of myofascial pain. J Indian Prosthodont Soc 2016;16:176-180.

21. Okeson JP. Management of Temporomandibular Disorders and Occlusion. St. Louis: Elsevier Health Sciences; 2014.

22. Armijo-Olivo S, Pitance L, Singh V, Neto F, Thie N, Michelotti A. Effectiveness of Manual Therapy and Therapeutic Exercise for Temporomandibular Disorders: Systematic Review and Meta-Analysis. Physical therapy. 2016; 96:9-25.

23. Suvinen T, Reade P. Prognostic features of value in the management of temporomandibular joint pain-dysfunction syndrome by occlusal splint therapy. J. Prosthet. Dent. 1989;61:355-61.

24. Tsuga K, Akagawa Y, Sakaguchi R, Tsuru H. A short-term evaluation of the effectiveness of stabilization-type occlusal splint therapy for specific symptoms of temporomandibular joint dysfunction syndrome. J Prosthet Dent 1989;61:610-3.

25. Truelove E, Huggins KH, Mancl L, Dworkin SF. The efficacy of traditional, low-cost and nonsplint therapies for temporomandibular disorder: A randomized controlled trial. J Am Dent Assoc 2006;137:1099-107.

26. Davies SJ, Gray RJ. The pattern of splint usage in the management of two common temporomandibular disorders. Part II: The stabilisation splint in the treatment of pain dysfunction syndrome. Br Dent J 1997;183:247-51.

27. Pettengill CA, Growney MR Jr, Schoff R, Kenworthy CR. A pilot study comparing the efficacy of hard and soft stabilizing appliances in treating patients with temporomandibular disorders. J Prosthet Dent 1998;79:165-8.

28. Block SL, Apfel M., Laskin DM. The use of a resilient rubber bite appliance in the treatment of MPD syndrome. J. Dent. Res. 1978;57:92. 